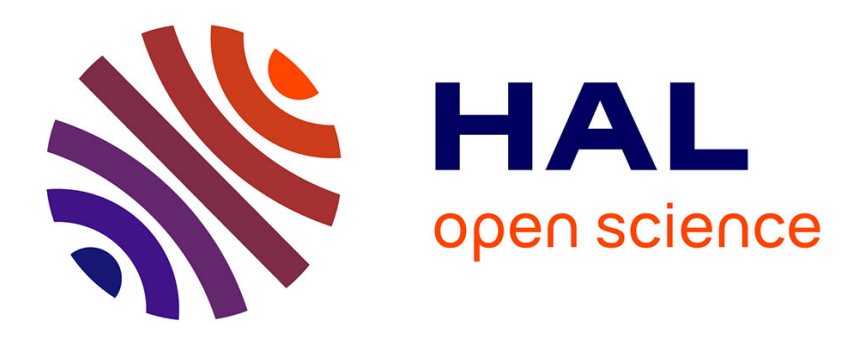

\title{
Vers une approche systémique des marques d'usage
}

Alain Polguère

\section{To cite this version:}

Alain Polguère. Vers une approche systémique des marques d'usage. Marques de registre dans les dictionnaires bilingues, Presses de l'Inalco, pp.3-32, 2020. hal-03070515

\section{HAL Id: hal-03070515 https://hal.science/hal-03070515}

Submitted on 15 Dec 2020

HAL is a multi-disciplinary open access archive for the deposit and dissemination of scientific research documents, whether they are published or not. The documents may come from teaching and research institutions in France or abroad, or from public or private research centers.
L'archive ouverte pluridisciplinaire HAL, est destinée au dépôt et à la diffusion de documents scientifiques de niveau recherche, publiés ou non, émanant des établissements d'enseignement et de recherche français ou étrangers, des laboratoires publics ou privés. 
Polguère, Alain (2020). Vers une approche systémique des marques d'usage. Dans Gueorgui Armianov (dir.) : Marques de registre dans les dictionnaires bilingues, série « Europe(s) », Paris : Presses de 1'Inalco, 3-32.

Nous définissons la marque d'usage comme étant une étiquette que le lexicographe attribue à une lexie pour signaler une contrainte sur l'emploi de cette dernière. La nature linguistique de cette contrainte se caractérise de façon négative : tout en étant intrinsèquement grammaticale (elle correspond à une règle linguistique générale), elle n'est ni sémantique, ni syntaxique, ni morphologique, ni phonologique. Après avoir postulé la nature grammaticale des marques d'usage, nous présentons l'approche adoptée pour leur utilisation dans deux projets lexicographiques issus de la Lexicologie Explicative et Combinatoire (LEC) : le Lexique actif du français (LAF) et le Réseau Lexical du Français.

Mots-clés : marque d'usage, Lexicologie Explicative et Combinatoire (LEC), Lexique actif du français (LAF), Réseau Lexical du Français, Système Lexical

We define a usage note as a label assigned by lexicographers to lexical units in order to indicate a given constraint on their use by speakers. The linguistic nature of this constraint in negatively characterized: while being intrinsically grammatical (it corresponds to a general linguistic rule), it is neither semantic, nor syntactic, nor morphological nor phonological. First, we postulate the grammatical nature of usage notes, after which we present the approach taken in using usage notes in two lexicographic projects based on Explanatory Combinatorial Lexicology (ECL): the Lexique actif du français 'Active French lexicon' (LAF) and the French Lexical Network.

Keywords: usage note, Explanatory Combinatorial Lexicology (ECL), Lexique actif du français (LAF), French Lexical Network, Lexical System

Под стилистической пометой понимается этикетка, которую лексикограф присваивает лексеме, чтобы обозначить накладываемые на ее употребление ограничения.

Лингвистическая природа этого ограничения характеризуется через отрицание - будучи по сути грамматическим (оно соответствует общему языковому правилу) оно не является ни семантическим, ни синтаксическим, ни морфологическим, ни фонологическим. Установив грамматическую природу помет, мы представим возможность их применения в двух лексикографических проектах в рамках Толково-комбинаторной лексикологии : Активная лексика франиузского языка и Лексическая сеть франиузского языка.

Ключевые слова: стилистические пометы, Толково-комбинаторная лексикология, Активная лексика франиузского языка, Лексическая сеть франиузского языка, лексическая система 


\section{Vers une approche systémique des marques d'usage}

Alain Polguère (Université de Lorraine, CNRS, ATILF)

\section{1. À propos de la notion de marque d'usage}

Nous entendons par marque d'usage une étiquette utilisée dans des modèles lexicographiques (dictionnaires ou bases de données lexicales) pour indiquer qu'une lexie donnée L possède une caractéristique $\mathrm{C}$ ayant les trois propriétés suivantes :

1. le choix de la lexie $\mathrm{L}$ dans certains contextes d'énonciation peut être inapproprié du fait que L possède la caractéristique $\mathrm{C}$;

2. la caractéristique $\mathrm{C}$ est, de ce fait, une contrainte dont le Locuteur $^{1}$ doit tenir compte dans son choix de $L$ ou d'une autre lexie (quasi-)synonyme pour exprimer le sens ' $L$ ' dans un énoncé donné2;

3. C n'est pas une caractéristique structurale de L, c'est-à-dire que c'est une caractéristique qui n'est ni sémantique, ni syntaxique, ni morphologique, ni phonologique.

Prenons le cas de fam ('familier') pour illustrer le type de caractéristique lexicale identifié par les marques d'usage. Associer dans un modèle lexical la marque d'usage fam à la lexie BOUFFER [Elle bouffe un sandwich.] :

1. signale qu'il pourrait être inapproprié pour le Locuteur d'utiliser BOUFFER dans certains contextes d'énonciation du fait qu'il s'agit d'une lexie appartenant à la langue familière ;

2. signale que le Locuteur doit tenir compte de la caractéristique ci-dessus lorsqu'il choisit BOUFFER dans un contexte donné d'énonciation pour exprimer le sens 'manger' ;

3. mentionne une caractéristique de BOUFFER qui n'est ni sémantique, ni syntaxique, ni morphologique, ni phonologique.

Le troisième paramètre de spécification de la caractéristique $\mathrm{C}$ dénotée par une marque d'usage est formulé négativement, car il est plus facile et rigoureux de dire ce que ne recouvre pas une marque d'usage que d'essayer d'énumérer tout ce qu'elle peut recouvrir. Il est fondamental que la marque d'usage identifie une contrainte d'emploi indépendante des caractéristiques structurales de la lexie qu'elle étiquette, car celles-ci sont prises en charge par d'autres éléments de la description lexicographique. Ainsi, il pourrait être inapproprié dans certains contextes d'énonciation d'utiliser la lexie VULVE - par exemple, au cours d'un dîner officiel avec des convives un peu prudes ou dans un article de linguistique. Cependant, ce fait est directement hérité du sens même de la lexie en question, qui est sa propriété structurale fondamentale : il est malvenu de parler d'organes génitaux dans certains contextes. Nul n'est besoin de marque d'usage pour identifier ce fait : la définition de VULVE suffit et fournit au Locuteur toute l'information dont il a besoin pour savoir s'il peut ou non faire usage de cette lexie, en l'absence justement de toute marque d'usage. (Il s'agit d'une lexie qui n'est ni familière, ni vulgaire, ni technique, etc.). Ce qui vient d'être dit à propos des propriétés sémantiques vaut pour toutes les autres propriétés structurales. Ainsi, l'indication qu'un nom donné est du genre féminin relève d'une contrainte sur la combinatoire de la lexie, mais cette contrainte

1 Nous écrivons Locuteur, avec une majuscule initiale, pour désigner la personne produisant un énoncé donné - à distinguer de locuteur d'une langue, écrit avec une minuscule initiale.

2 Cette contrainte s'applique aussi dans le cadre de l'implémentation du choix lexical en Traitement Automatique de la Langue, notamment en génération de texte ou en traduction automatique (DiMarco et Hirst, 1993). 
étant liée au comportement syntactico-morphologique de la lexie dans la phrase, la mention « fém » ('genre grammatical féminin') dans un article lexicographique ne relève pas de la marque d'usage.

Une marque d'usage n'est pas nécessairement une marque de registre, au sens où l'on entend généralement ce terme. Par exemple, néol ('néologisme') est une marque d'usage, qui répond aux trois critères énoncés ci-dessus, mais elle n'identifie pas l'appartenance d'une lexie à un registre de langue donné. Dans le présent exposé, nous choisissons de nous intéresser aux marques d'usage au sens large, plutôt que de nous concentrer sur les seules marques de registre, si l'on prend la notion de registre (angl. register) dans son interprétation hallidayienne de variété fonctionnelle d'une langue (Halliday et Matthiessen, 2004). En effet, notre but est de présenter un modèle général, intégrant toutes les contraintes d'emploi des lexies répondant aux trois critères ci-dessus, la contrainte identifiée pouvant relever d'une variété fonctionnelle de la langue (par exemple, langue familière) aussi bien que d'une caractéristique individuelle d'une lexie portant, par exemple, sur son historique d'apparition dans la langue (comme dans le cas des néologismes).

Notre cadre théorique et descriptif de référence est la Lexicologie Explicative et Combinatoire ou LEC (Mel'čuk et coll., 1995), la composante lexicale de la théorie linguistique Sens-Texte (Mel'čuk, 1997). Après avoir postulé la nature grammaticale des marques d'usage (section 2), nous présenterons l'approche adoptée pour leur utilisation dans deux projets lexicographiques de la LEC : le Lexique Actif du Français (section 3) et le Réseau Lexical du Français (section 4).

Les modèles lexicaux construits dans le cadre de ces projets sont monolingues et l'on sait que la problématique des marques d'usage est particulièrement cruciale dans le contexte de la lexicographie multilingue ${ }^{3}$. Notons cependant que la LEC adopte la perspective de l'encodage dans l'élaboration de ses modèles lexicographiques, perspective qui est au cœur de la lexicographie multilingue et, plus généralement, de la lexicographie visant la construction de dictionnaires d'apprentissage (Bogaards, 1996).

\section{Les marques d'usage en tant que caractéristiques grammaticales}

Dire que les marques d'usage n'identifient pas de propriétés structurales des lexies qu'elles étiquettent ne signifie pas qu'elles ne sont pas grammaticales par nature. Si l'on considère que la grammaire est le module de la langue qui contient ses règles générales ${ }^{4}$, force est de constater qu'une règle présidant à l'usage ou au non-usage de lexies familières, néologiques, etc., dans des contextes d'énonciation donnés est une règle tout à fait générale - de bienséance ou d'efficacité linguistique -, qui peut être formulée sans mentionner de lexies spécifiques.

Nous considérons donc que les marques d'usage rendent compte d'un cas particulier, assez marginal, de caractéristiques grammaticales des lexies, qui cohabitent notamment avec leurs caractéristiques structurales, tout en formant une classe à part.

Cette intégration des marques d'usage dans les caractéristiques grammaticales - entendues au sens large - nous semble nécessaire pour deux raisons. Tout d'abord, elle est légitime et découle logiquement de la conceptualisation de la grammaire en tant que module de règles linguistiques générales (non formulées relativement à des lexies données). Ensuite, elle apporte un remède à la façon traditionnelle de concevoir les marques d'usage, qui se retrouvent un peu dans la microstructure des dictionnaires de langue comme des parias, sans statut clair vis-à-vis des trois grands axes de caractérisation des lexies, hérités de la structure même du signe linguistique : sens, forme et combinatoire

$3 C f$. la thématique du volume dans lequel le présent article est publié.

4 « [G]rammar deals with the general facts of language, and lexicology with special facts » (Jespersen, 1924 : 32). On notera aussi que dans l'approche de la linguistique systémique, déjà mentionnée plus haut (Halliday et Matthiessen, 2004), les systèmes présidant aux choix linguistiques typiquement associés aux marques d'usage - comme ceux concernant le registre de langue - font partie intégrante de la lexico-grammaire (lexicogrammar) de la langue. Le choix de registre est de ce fait un choix grammatical. 
restreinte (Mel'čuk, 1993 : Chapitre II). Les marques d'usage relèvent de la caractérisation de la combinatoire restreinte des lexies et sont éminemment grammaticales : associer une marque d'usage à une lexie donnée revient à encoder une règle lexicale, mais les règles qui spécifient comment employer les lexies portant la marque d'usage en question sont, elles, parfaitement générales, donc grammaticales.

Pour conclure sur ce sujet, notons que la caractérisation en trois points présentée dans la section 1 vaut pour les marques d'usage lexicographiques, appliquées à des lexies. Dans la pratique, une marque d'usage peut aussi tout à fait s'appliquer à n'importe quelle entité linguistique, y compris une entité grammaticale comme une règle syntaxique ou morphologique. Ainsi, la règle de sélection du passé simple en français contemporain doit être étiquetée par une marque d'usage, puisque l'emploi de ce temps grammatical est stylistiquement marqué, par opposition à celui du passé composé. La même remarque s'applique à certains types de pronominalisations relatives (le gars que je sors avec). La caractérisation de la section 1 pourrait donc être élargie aux contraintes d'usage de toute entité linguistique (lexicale ou grammaticale).

\section{Typologie des marques d'usage dans le Lexique actif du français (LAF)}

Le Lexique actif du français, désormais $L A F$ (Mel'čuk et Polguère, 2007), est un ouvrage à visée didactique pour lequel a été élaboré, pour la première fois dans le contexte de la LEC, une classification des marques d'usage. Cette dernière a été reprise par la suite dans les travaux sur les Systèmes Lexicaux, présentés à la section 4. Nous ferons d'abord une brève introduction au LAF : sa finalité et son contenu (3.1). Nous présenterons ensuite le système de marques d'usage qui a été utilisé pour sa réalisation et qui repose sur une typologie relativement simple en trois classes principales (3.2).

\subsection{Outil pédagogique fondé sur l'exploitation des fonctions lexicales Sens-Texte}

Le LAF est destiné notamment à aider les enseignants dans l'élaboration d'activités d'enseignement du vocabulaire (Piémont, 2008), avec une focalisation sur l'exploration des liens lexicaux paradigmatiques fondamentaux, appelés dérivations sémantiques, et des liens lexicaux syntagmatiques qui unissent une base à ses collocatifs au sein d'expressions semi-phraséologiques - les collocations au sens de Hausmann (1979). L'ouvrage se divise en deux parties complémentaires. La première partie est de nature théorique et introduit notamment les notions fondamentales d'unité lexicale (ou lexie), vocable polysémique (regroupement de lexies), dérivation sémantique, collocation, etc. Cette partie présente aussi la structure et le mode d'utilisation de la seconde partie, le LAF proprement dit, qui est un échantillon de dictionnaire du français, dont chaque article décrit la lexie vedette en termes des liens lexicaux paradigmatiques et syntagmatiques qu'elle contrôle. La figure 1 ci-dessous reproduit l'entrée DÉFAITE du LAF'.

5 Nous avons sélectionné cet article, parce qu'il est relativement compact ; la plupart des entrées du LAF sont bien plus développées, notamment parce qu'elles décrivent des vocables polysémiques. L'article de la Figure 1 présente de plus l'avantage de contenir plusieurs occurrences d'une marque d'usage (fam). 
DÉFAITE, nom, fém

\begin{abstract}
ÉVÉNEMENT NON SOUHAITABLE
Défaite de la personne $\mathrm{X}\left[=\right.$ de $\mathrm{N}, \mathrm{A}_{\mathrm{poss}}$, Adj (ex. défaite française)] face à la personne $\mathrm{Y}\left[=\left\ulcorner^{\ulcorner}\right.\right.$face $\grave{a}^{\urcorner} \mathrm{N}$, litt $\left\ulcorner^{\ulcorner}\right.$aux mains $d e^{\urcorner} \mathrm{N}$ ] dans la confrontation $\mathrm{Z}$ $[=$ dans $\mathrm{N}]$

échec; fam déconfiture, fam piquette

Ant. victoire I Verbe perdre [contre $\mathrm{N}_{\mathrm{Y}}$ ] Nom pour $\mathrm{X}$ perdant ${ }_{\mathrm{N}}$, vaincu $\mathrm{N}_{\mathrm{N}}$ Nom pour $Y$ gagnant $_{N}$, vainqueur ${ }_{N}$ Qui est $X$ dans une $D$. perdant ${ }_{A d j}$, vaincu $_{A d j}$; défait Qui est $Y$ dans une $D$. gagnant ${ }_{A d j}$, vainqueur ${ }_{A d j}$, victorieux Grave grande, grosse, lourde | antépos, grave, sévère, sérieuse < écrasante, terrible < complète, totale // débâcle, déroute, fam raclée Grave et qui fait moralement du mal à $X$ cuisante, honteuse, humiliante Qui s'accompagne de la mort de nombreux $X$, pour une défaite militaire sanglante Qui va se produire certaine $\mid$ postpos, fatale, inévitable $[X]$ subir une D. connaître, fam encaisser, essuyer, subir [ART $\sim{ }^{\ulcorner}$face à ${ }^{\urcorner} N_{Y}$ dans $N_{Z}$ ] [X] être sur le point de subir une D. s'acheminer [vers ART $~<$ courir [à la $\sim] /[$ une $\sim$ | avec modificateur] [Y] faire subir une D. à Y faire subir, infliger $\left[\mathrm{ART} \sim\right.$ à $\mathrm{N}_{\mathrm{X}}$ ]

Après la défaite allemande, il fit ses études théologiques au Grand séminaire de Cracovie. L'ancien maire est déçu, mais, comme lors de sa défaite aux municipales, il se montre bon perdant. La III République est proclamée à la suite de la défaite de Sedan.
\end{abstract}

Figure 1 : Article lexicographique de DÉFAITE dans Mel'čuk et Polguère (2007: 186)

Comme on le voit, les articles du LAF ne contiennent pas de définitions lexicographiques. Celles-ci sont remplacées par un étiquetage sémantique des lexies vedettes (Polguère, 2011) et par la spécification de leur structure actancielle - la référence aux positions actancielles $(X, Y, Z)$ joue en effet un rôle clé dans le corps de l'article lexicographique. Les liens lexicaux sont caractérisés par des formules (en gras), qui en explicitent la valeur sémantico-syntaxique. Ces formules sont appelées formules de vulgarisation, car elles correspondent à une paraphrase en français contrôlé de l'encodage des liens lexicaux au moyen du formalisme des fonctions lexicales de la théorie SensTexte (Mel'čuk, 1996). Pour ce qui est de sa méthodologie d'élaboration, le LAF a été manuellement produit en effectuant la traduction sous forme de dictionnaire grand public de l'information formellement encodée dans la base de données lexicale DiCo (Polguère, 2000). Ainsi, la section de l'article de DÉFAITE ci-dessus qui introduit les dérivés sémantiques PERDANT Adj, VAINCU $_{\text {Adj }}$ et DÉFAIT provient des données suivantes du DiCo, où les noms de fonctions lexicales identifiant les liens paradigmatiques en question sont mentionnés entre accolades $\{\ldots\}$, précédés de leur formule de vulgarisation balisée par $/ * \ldots * /$ :

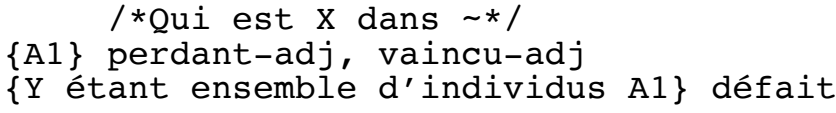

Le DiCo peut être considéré comme une incarnation informatisée des principes de la LEC destinée aussi bien à la génération d'articles lexicographiques, tels que ceux du LAF, qu'à une exploitation informatique dans un cadre de Traitement Automatique de la Langue.

Les marques d'usage jouent un rôle important dans le LAF, car elles permettent d'orienter les choix lexicaux parmi les différentes valeurs proposées pour un lien lexical donné. Bien entendu, les marques d'usage étiquettent aussi directement les lexies vedettes. Ainsi, la marque d'usage fam, qui étiquette dans la Figure 1 l'occurrence de déconfiture en tant que quasi-synonyme de la lexie vedette (symbole $)$, doit étiqueter le lexème correspondant dans son article lexicographique. Attention cependant : cela n'est pas systématique et la cible d'un lien lexical du LAF (ou du DiCo) peut être étiquetée par une marque d'usage, sans que la lexie correspondante le soit dans son article lexi- 
cographique. Tel est le cas de nombre de cibles de liens syntagmatiques, où l'emploi d'une lexie en tant que collocatif de la lexie vedette base de la collocation est marqué, même si le collocatif en question est d'un emploi tout à fait neutre dans des expressions libres (non phraséologiques), comme l'illustre l'extrait de l'article ABCÈS I du LAF donné ci-dessous (Mel'cuk et Polguère, $2007: 88)$ :

\section{Sans inflammation spéc froid}

Ce n'est pas l'adjectif froid qui relève du langage spécialisé (de la médecine), mais la collocation abcès froid au complet qui fonctionne comme un terme.

Avant le travail mené sur le LAF, les marques d'usage ne possédaient pas de statut théorique spécifique dans la LEC; elles sont ainsi employées sans véritable systématisation dans les quatre volumes publiés du Dictionnaire Explicatif et Combinatoire ou DEC (Mel'čuk, 1994-1988-19921999). Elles sont simplement énumérées dans la section présentant les Notations utilisées. Les marques d'usage apparaissent dans les entrées de DEC avec les autres caractéristiques grammaticales des lexies (partie du discours, genre pour les noms, etc.). On notera que la nature grammaticale des marques d'usage est reconnue dès la publication du premier volume du DEC, en 1984, même si le choix du terme syntaxique plutôt que grammatical, pour les caractériser, est un peu malheureux : «Une marque d'usage, étant considérée comme l'expression d'une particularité syntaxique au sens large du terme, est mise dans cette division ${ }^{6}$, à la suite des autres marques syntaxiques. » (Elnitsky, $1984: 19)$.

La même approche un peu nonchalante vis-à-vis des marques d'usage a été appliquée dans le projet du DiCo. Il a cependant semblé impossible de ne pas rationaliser le système de marques d'usage dans le cadre d'un ouvrage à caractère didactique tel que le LAF et son élaboration a ainsi permis d'équiper un peu mieux la LEC sur ce sujet qu'elle avait délaissé. Nous allons maintenant présenter la classification générale des marques d'usage élaborée pour le LAF.

\subsection{Classification générale}

Les auteurs du LAF ont décidé de regrouper les marques d'usage utilisées dans l'ouvrage - 17 marques d'usage distinctes - en trois grandes classes, fondées sur le type de choix effectué par le Locuteur lorsqu'il doit lexicaliser son message ${ }^{7}$. Le patron terminologique utilisé pour nommer chacune des trois classes est le suivant :

Marque d'usage + Adj (où Adj est un modificateur syntaxique de $u s a g e^{8}$ ).

Nous allons présenter tour à tour les trois classes en question :

- marques d'usage langagier (3.2.1);

- marques d'usage stylistique (3.2.2);

- marques d'usage rhétorique (3.2.3).

6 Il s'agit de la division de la microstructure des DEC qui contient les informations dites « syntaxiques » sur la lexie vedette et qui suivent les informations " morphologiques » (partie du discours, genre, etc.). L'exemple donné dans le texte cité ci-dessus est : « PATHOGĖNE, adj, postpos, spéc. ».

7 Les trois classes de marques d'usage sont introduites à la p. 60 de la première partie du LAF. Chaque marque d'usage est ensuite individuellement caractérisée au chapitre 4 (Abréviations, conventions et symboles) de la même partie.

8 En d'autres termes, l'adjectif Adj identifiant la classe de marques d'usage est au masculin singulier. C'est l'usage qui est Adj et non la/les marque(s). 


\subsubsection{Marques d'usage langagier}

Les marques d'usage langagier dénotent des contraintes liées aux propriétés du code linguistique lui-même : paramètres historiques, géographiques, etc. On peut considérer qu'il s'agit de contraintes objectives dans la mesure où, contrairement aux contraintes dénotées par les deux autres classes de marques d'usage présentées plus bas, elles ne concernent pas une perspective communicative adoptée par le Locuteur dans la construction de son énoncé. Le LAF a recours à cinq marques d'usage langagier, décrites ci-dessous.

- angl 'anglicisme' (par exemple, black-out dans l'article de SILENCE I.2b) : Il s'agit d'une lexie ou expression considérée comme provenant de l'anglais, qui est un emprunt plutôt récent à cette langue, ou qui est perçu comme tel. Son usage peut être condamné par les personnes ayant une attitude normative vis-à-vis de la langue.

Il est important de noter qu'une lexie peut en théorie être étiquetée angl même si elle n'est pas un emprunt de l'anglais, du moment qu'elle tend à être perçue comme provenant de cette langue et comme n'étant pas complètement intégrée au système du français. La même logique s'applique à toutes les marques d'usage langagier, qui n'ont pas pour fonction d'encoder la réalité historique, géographique, etc., mais bien une perception courante chez les locuteurs du français.

- néol 'néologisme' (par exemple, sandwicherie dans l'article de SANDWICH) ${ }^{9}$ : Un néologisme est perçu comme étant apparu récemment en français, et comme n'étant pas un emprunt - sinon, une marque d'usage du type angl s'applique et suffit. Son usage peut être perçu comme condamnable.

- rare (par exemple, exécration dans l'article de HAINE) : On entend par terme rare un terme rarement employé parce que peu connu des locuteurs. L'utilisation de cette marque d'usage dans le LAF est fondée sur une perception tout à fait subjective des lexicographes et de leurs informateurs, qui ne repose pas sur des évidences statistiques extraites de l'analyse de corpus. Il n'existe pas à l'heure actuelle, selon nous, de corpus du français suffisamment fiables, vastes et représentatifs pour pouvoir fonder l'attribution de ce type de marque d'usage sur des critères purement statistiques. Quoi qu'il en soit, la marque d'usage rare est nécessairement problématique et doit être utilisée avec une grande modération.

- spéc 'qui relève d'une ou plusieurs langue(s) de spécialité' (par exemple, actinite comme quasi-synonyme dans l'article de COUP DE SOLEIL) : Cette marque d'usage identifie l'entité lexicale en question comme relevant du langage technique, scientifique et, plus généralement, spécialisé. Elle a donc avant tout sa place dans des textes spécialisés : domaines de l'informatique, de la médecine, de la chimie, de la religion, etc. Le LAF ne possède qu'une marque d'usage pour les terminologies, contrairement à nombre de dictionnaires et ressources terminologiques, qui ont recours à l'identification de domaines (info, milit, relig, etc.) et, même, de sous-domaines. Nous aurons l'occasion d'expliquer pourquoi il est procédé de la sorte, lorsque nous traiterons des marques d'usages dans les Systèmes Lexicaux (section 4). Notons d'ores et déjà que cet étiquetage minimal des termes est en accord avec une volonté de limiter au maximum la palette des marques d'usage utilisée dans les modèles lexicographiques de la LEC.

- vieilli 'vieilli ou vieillissant' (par exemple, pneumatique comme quasi-synonyme dans l'article de PNEU) : Il s'agit de lexies ou expressions qui, sans être obsolètes, sont d'un usage plutôt associé au français parlé par les générations précédentes ${ }^{10}$.

9 La lexie SANDWICHERIE pouvait être considérée comme néologique au moment de la rédaction du LAF. Ce n'est peut-être plus le cas, douze ans plus tard. 


\subsubsection{Marques d'usage stylistique}

Les marques d'usage stylistiques donnent une indication sur le contexte socioculturel, au sens large, associé à l'emploi de l'entité lexicale étiquetée. Les articles de LAF ont recours à neuf marques d'usage de ce type, que nous allons nous contenter d'énumérer, car leur emploi dans cet ouvrage ne présente pas d'originalité particulière :

- enfantin (par exemple, tagada dans l'article de GALOP);

- fam 'familier' (par exemple, le vocable ENGUEULADE);

- formel (par exemple, l'expression Nous avons le regret de vous annoncer la mort de $X$ dans l'article de MORT $\left.{ }^{1} \mathbf{I} .1\right)$;

- litt 'littéraire' (par exemple, être paré dans l'article de PLUMAGE 1);

- offic 'officiel' (par exemple, avertisseur sonore dans l'article KLAXON a) - emploi caractéristique du langage administratif, légal, etc. ;

- relig 'religieux' (par exemple, l'expression en état péché dans l'article de PÉCHÉ);

- rural (par exemple, bestiaux dans l'article de BÉTAIL) - lexie ou expression associée au parler des campagnes ;

- soutenu (par exemple, faconde dans l'article de CONVERSATION b) ;

- vulg 'vulgaire' (par exemple, couille dans l'article de TESTICULE).

Il faut noter qu'il n'est pas toujours aisé de déterminer quand utiliser les marques d'usage stylistique offic et relig, plutôt que la marque d'usage langagier spéc. On pourra ainsi considérer qu'une lexie comme CATÉCHUMÈNE doit être étiquetée spéc, car elle relève de la terminologie religieuse (chrétienne), typiquement employée par un membre du clergé. L'expression en état de péché, par contraste, peut être considérée comme non terminologique et comme possédant simplement une coloration stylistique propre à la façon de s'exprimer des croyants. Nous devons avouer cependant que nous ne sommes pas convaincu de la véracité de ce que nous venons d'écrire et que nous sommes très tenté par l'éradication des marques d'usage du type offic et relig au profit de la seule marque d'usage langagier spéc.

\subsubsection{Marques d'usage rhétorique}

Les marques d'usage rhétorique signalent une perspective adoptée par le Locuteur vis-à-vis des différents paramètres de la situation d'énonciation. Elles nous informent que l'emploi de la lexie étiquetée implique nécessairement l'expression par le Locuteur d'un point de vue affectif, évaluatif, idéologique, etc., quant à la chose dénotée. Le LAF a recours aux trois marques d'usage rhétorique suivantes :

10 Comme nous l'a fort justement fait remarquer un relecteur anonyme d'une version antérieure du présent texte, pneumatique est d'un usage courant dans le domaine de la compétition automobile, notamment en Formule 1 (F1). Le LAF aurait donc dû faire usage d'une marque d'usage double disjonctive : vieilli ou spéc. Une alternative est de considérer qu'il existe une acception particulière du vocable nominal PNEUMATIQUE ${ }_{(\mathrm{N})}$ qui relève du domaine spécialisé de la course automobile. Cette acception aurait donc la marque d'usage spéc et l'acception plus vague non spécialisée garderait sa marque d'usage simple vieilli. 
- affectueux (par exemple, la lexie POULE III [Comment ça va, ma poule ?]) ${ }^{11}$;

- iron 'ironique' (par exemple, le collocatif intensificateur cordialement dans l'article de HAÏR) ;

- péjor 'péjoratif' (par exemple, vieille fille dans l'article de CÉLIBATAIRE).

Nous allons maintenant examiner comment le système de marques d'usage du LAF a été utilisé dans le cadre d'un récent projet lexicographique fondé sur la construction, non de dictionnaires, mais de réseaux lexicaux appelés Systèmes Lexicaux, en nous intéressant tout particulièrement au cas de la marque d'usage langagier spéc.

\section{Perspective relationnelle dans les Systèmes Lexicaux}

La construction d'un nouveau type de modèle lexicographique a récemment été entamée dans le cadre de la LEC autour du projet du Réseau Lexical du Français (Lux-Pogodalla et Polguère, 2011). Il s'agit non plus de construire un dictionnaire, c'est-à-dire un modèle textuel du lexique, mais de tisser un Système Lexical, dont la structure de graphe est fondée sur les fonctions lexicales paradigmatiques et syntagmatiques Sens-Texte (Polguère, 2014). La lexicographie des Systèmes Lexicaux relève fondamentalement de la pratique de la LEC, mais le recours à un modèle lexical en réseau marque une coupure assez radicale dans la pratique lexicographique traditionnelle. Ainsi, décrire une lexie dans un Système Lexical ne se fait pas en écrivant un article lexicographique, mais en créant un nouveau nœud dans le réseau lexical de la langue et en le connectant aux autres nœuds du réseau via le tissage de multiples liens de fonctions lexicales paradigmatiques et syntagmatiques. De plus, chaque nœud lexical est enrichi d'informations grammaticales, dont justement les marques d'usage, par l'assignation de traits correspondants : tissage d'un lien grammatical entre la lexie caractérisée et un élément spécifique du métamodèle grammatical (marque d'usage, partie du discours, etc.).

Le projet du Réseau Lexical du Français a adopté la typologie des marques d'usage introduite dans le LAF, en modifiant et enrichissant la palette des marques d'usage au fur et à mesure que de nouveaux cas de figure étaient rencontrés dans le travail lexicographique. Le développement des marques d'usage se fait donc de façon inductive, à partir de l'hypothèse initiale que représente la typologie en trois classes principales postulées dans le LAF (section 3.2). Nous présentons en Annexe le système actuel des marques d'usage du Réseau Lexical du Français, tel qu'il se visualise dans l'éditeur lexicographique employé dans ce projet lexicographique (Gader et coll., 2012).

Dans un Système Lexical, chaque lexie peut être considérée comme contrôlant des espaces sémantiques ou, plus généralement, des espaces lexicaux, déterminés par la topologie du graphe lexical. On peut ainsi caractériser une lexie en regard de la proximité topologique relative d'autres lexies de la langue. C'est cette propriété du modèle des Systèmes Lexicaux qui va nous intéresser tout particulièrement ici. Nous voudrions en effet revenir sur le cas de la marque d'usage langagier spéc (section 3.2.1) et sur la décision qui a été prise dans notre approche de n'avoir recours qu'à cette marque d'usage unique, plutôt que de développer un ensemble de marques identifiant des domaines (et sous-domaines) de spécialité, comme le font la plupart des modèles lexicographiques et terminographiques.

Nous faisons les trois critiques suivantes de l'approche consistant à développer un système de marques d'usage de domaines terminologiques.

1. Le système en question est ouvert et peut se complexifier à l'infini, selon l'évolution des techniques, des sciences et, plus généralement, des domaines d'activités. Il est de plus im-

11 Nous ajouterions maintenant dans l'article de la lexie POULE III une seconde marque d'usage rhétorique, disponible dans la palette des marques d'usage du Réseau Lexical du Français (cf. section 4 et Annexe) : peut être offensant. 
possible d'établir des critères linguistiques et rigoureux de détermination des domaines en question, les critères variant selon les besoins individuels de classification.

2. L'appartenance à un ou plusieurs domaines et sous-domaines de spécialité est une conséquence directe de la dénotation de la lexie et est déductible de sa définition ainsi que des liens paradigmatiques qui la connectent à d'autres lexies de la langue. Ainsi, le Petit Robert (2017) étiquette la lexie SERVEUR 3 [Appuyez sur la touche "Entrée " pour redémarrer le serveur.] par la marque d'usage «INFORM. », alors que l'article lexicographique nous indique clairement qu'il ne s'agit bien évidemment pas d'un terme de zoologie, de médecine ou d'agriculture : SERVEUR 3 est défini par «Système informatique chargé de fournir des services, des informations à d'autres systèmes, à des usagers connectés » et mentionne des termes comme base de données et Internet. Il serait donc amplement suffisant d'indiquer à l'usager du dictionnaire que SERVEUR 3 est de nature terminologique, le domaine technique dont relève ce terme étant une évidence.

3. Les domaines de spécialités associés à un terme doivent être hérités du système de connexions lexicales qui relie le terme en question aux lexies de la langue, ce qui permet à un terme d'appartenir simultanément et à des degrés divers à plusieurs domaines. La prédétermination des domaines de spécialité est donc non seulement une tâche lexicographique qui débouche sur des descriptions très contestables, mais elle est surtout une tâche inutile : on peut générer à la demande un ensemble de domaines associés à un terme par simple analyse manuelle ou automatique des informations relationnelles présentes dans le modèle lexicographique.

Pour illustrer cela, prenons le cas de la lexie CRÉPINE [Voilà la recette des filets mignons de porc en crépine.], étiquetée spéc dans le Réseau Lexical du Français. Une analyse topologique du graphe lexical au moyen d'un algorithme dit de proxémie (Gaume, 2004) permet d'identifier plusieurs espaces sémantiques associables à CRÉPINE, qui sont visualisés dans la Figure 2 ci-dessous.

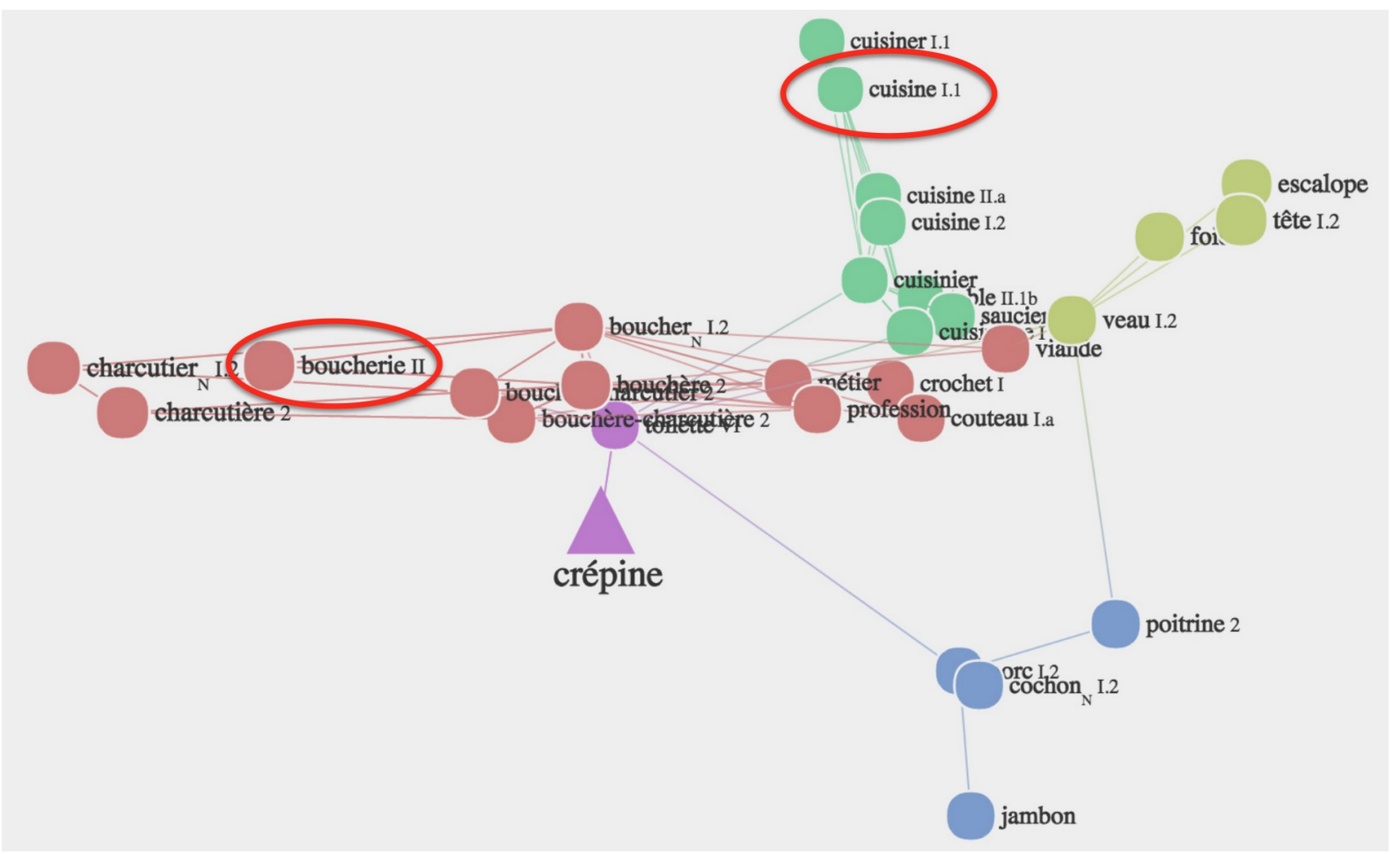

Figure 2 : Espace sémantique de spéc CRÉPINE dans le Réseau Lexical du Français

On voit ici apparaître deux unités lexicales - BOUCHERIE II et CUISINE I.1 - qui sont autant d'identificateurs de domaines terminologiques. L'association de ces identificateurs à CRÉPINE à par- 
tir de la structure même de l'information lexicale nous semble une méthode tout à la fois flexible, rigoureuse et efficace de caractérisation par domaines des unités terminologiques. Il suffit au lexicographe d'identifier une lexie comme n'appartenant pas à la langue générale et de lui appliquer en conséquence la marque d'usage langagier spéc pour signaler ce fait et avertir l'utilisateur du modèle de la nature terminologique de la lexie en question. Si une liste de domaines de spécialité s'avère nécessaire pour caractériser une lexie, elle peut être automatiquement déduite de l'information contenue dans le reste de l'information lexicographique et cette liste pourra évoluer avec l'évolution du modèle lexical dans son ensemble (changements dans la topologie du graphe lexical) ${ }^{12}$.

Nous nous sommes attardé, dans cette dernière section, sur le cas de la marque d'usage spéc, car la spécification des domaines de spécialité génère une grande activité en lexicographie et terminographie (Buvet et Mathieu-Colas, 1999), alors même que l'on peut vraisemblablement la contourner si l'on développe des modèles lexicographiques véritablement relationnels. Elle produit de plus une inflation de marques d'usage, qui va à l'encontre d'un principe fondamental d'économie qui devrait nous guider dans notre recours aux marques d'usage.

\section{Conclusions}

Nous avons présenté dans cet article la marque d'usage en tant que caractéristique grammaticale des lexies qui dénote des contraintes quant au choix de lexicalisation que peut opérer le Locuteur. Ces contraintes sont caractérisées négativement : elles sont non-structurales ; en d'autres termes, elles sont de nature ni sémantique, ni syntaxique, ni morphologique, ni phonologique. Cela permet, d'une part, de définir chaque marque d'usage en fonction du type de contraintes de lexicalisation auxquelles elle renvoie et, d'autre part, de regrouper les marques d'usage en trois classes relativement distinctes : marques d'usage langagier, stylistique ou rhétorique.

Dans le cadre de la Lexicologie Explicative et Combinatoire (LEC) telle que mise en pratique dans le Réseau Lexical du Français, les marques d'usage sont entièrement intégrées au système global des caractéristiques grammaticales (parties du discours, restrictions sur la flexion, etc.) au sein duquel elles font système. Elles ne forment donc pas, comme cela est généralement le cas dans les dictionnaires, un ensemble de traits déconnectés des autres éléments de caractérisation des lexies. De plus, elles sont conçues pour prendre leur valeur au sein de modèles relationnels appelés Systèmes Lexicaux. Cette valeur systémique des marques d'usage a été illustrée avec le cas particulier de la marque spéc : elle identifie uniquement une contrainte portant sur la nature technique, scientifique, etc., de la lexie qui la porte, sans qu'il soit besoin d'identifier des domaines et sous-domaines de spécialité spécifiques. Ces derniers peuvent être directement déduits du positionnement de la lexie dans le réseau lexical global de la langue : sa relative proximité topologique avec des lexies identificatrices de domaines et sous-domaines.

\section{Références bibliographiques}

BOGAARDS, P. 1996. Dictionaries for Learners of English. International Journal of Lexicography, 9(4), p. 277-320.

BUVET, P.-A., MATHIEU-COLAS, M. 1999. Les champs Domaine et Sous-domaine dans les dictionnaires électroniques. Cahiers de Lexicologie, 75, p. 173-191.

CASTILLO, M., REAL, F., RIGAU, G. 2004. Automatic Assignment of Domain Labels to WordNet. In P. Sojka, K. Pala, P. Smrž, C. Fellbaum et P. Vossen (dir.) : Proceedings of the Second International WordNet Conference (GWC 2004), Brno, Masaryk University, p. 75-82.

12 Pour une application d'une stratégie similaire sur la structure relationnelle de la base lexicale de l'anglais WordNet, voir Castillo et coll. (2004). 
DIMARCO, C., HIRST, G. 1993. Usage notes as the basis for a representation of near-synonymy for lexical choice (or Making words of senses). In : Proceedings of the $9^{\text {th }}$ annual conference of the University of Waterloo Centre for the New Oxford English Dictionary and Text Research, Oxford, September 1993, Oxford, p. 33-43.

ELNITSKY, L. 1984. Présentation d'un article de dictionnaire (lexème) et d'un superarticle (vocable), In I. Mel'čuk, N. Arbatchewsky-Jumarie, L. Elnitsky, L. Iordanskaja et A. Lessard (dir.) : Dictionnaire explicatif et combinatoire du français contemporain. Recherches lexico-sémantiques. Volume I, Montréal, Les Presses de l'Université de Montréal, p. 17-25.

GADER, N., LUX-POGODALLA, V., POLGUÈRE, A. 2012. Hand-Crafting a Lexical Network With a Knowledge-Based Graph Editor. In : Proceedings of the Third Workshop on Cognitive Aspects of the Lexicon (CogALex III), Mumbai, The COLING 2012 Organizing Committee, p. 109125.

GAUME, B. 2004. Balades aléatoires dans les Petits Mondes Lexicaux. I3 Information Interaction Intelligence, 4(2), p. 39-96.

HALLIDAY, M. A. K., MATTHIESSEN, C. M. I. M. 2004. An introduction to functional grammar, $3^{\mathrm{e}}$ édition, Londres, Hodder Arnold.

HAUSMANN, F. J. 1979. Un dictionnaire des collocations est-il possible ? Travaux de littérature et de linguistique de l'Université de Strasbourg, XVII(1), p. 187-195.

JESPERSEN, O. 1924. The Philosophy of Grammar, Londres, George Allen \& Unwin.

LUX-POGODALlA, V., POLGUÈRE, A. 2011. Construction of a French Lexical Network: Methodological Issues. In : Proceedings of the First International Workshop on Lexical Resources, WoLeR 2011. An ESSLLI 2011 Workshop, Ljubljana, p. 54-61.

MEL'ČUK, I. 1993. Cours de morphologie générale (théorique et descriptive). Volume I. Introduction et Première partie : Le mot, Montréal \& Paris, Les Presses de l'Université de Montréal \& CNRS Éditions.

MEL'ČUK, I. 1996. Lexical Functions : A Tool for the Description of Lexical Relations in the Lexicon, In L. Wanner (dir.) : Lexical Functions in Lexicography and Natural Language Processing, Language Companion Series 31, Amsterdam/Philadelphia, John Benjamins, p. 37-102.

MEL'ČUK, I. 1997. Vers une linguistique Sens-Texte. Leçon inaugurale, Paris, Collège de France.

MEL'ČUK, I., CLAS, A., POLGUÈRE, A. 1995. Introduction à la lexicologie explicative et combinatoire, Paris/Louvain-la-Neuve, Duculot.

MEL'ČUK, I., POLGUÈRE, A. 2007. Lexique actif du français. L'apprentissage du vocabulaire fondé sur 20000 dérivations sémantiques et collocations du français, Bruxelles, De Boeck \& Larcier. 
MEL'ČUK, I. et coll. 1984-1988-1992-1999. Dictionnaire explicatif et combinatoire du français contemporain. Recherches lexico-sémantiques. Volumes I-IV, Montréal, Les Presses de l'Université de Montréal.

PIÉMONT, N. 2008. Enseigner le lexique au collège. Approche didactique de la lexicologie explicative et combinatoire - https://www.scribd.com/doc/294701855/Piemont-2008, consulté le 7 juillet 2919.

POLGUÈRE, A. 2000. Towards a theoretically-motivated general public dictionary of semantic derivations and collocations for French. In : Proceedings of EURALEX'2000, Stuttgart, p. 517-527.

POLGUÈRE, A. 2011. Classification sémantique des lexies fondée sur le paraphrasage. Cahiers de lexicologie, 98, p. 197-211.

POLGUÈRE, A. 2014. From Writing Dictionaries to Weaving Lexical Networks. International Journal of Lexicography, 27(4), p. 396-418. 
Annexe :

Hiérarchie des marques d'usage du Réseau Lexical du Français (version juillet 2019)

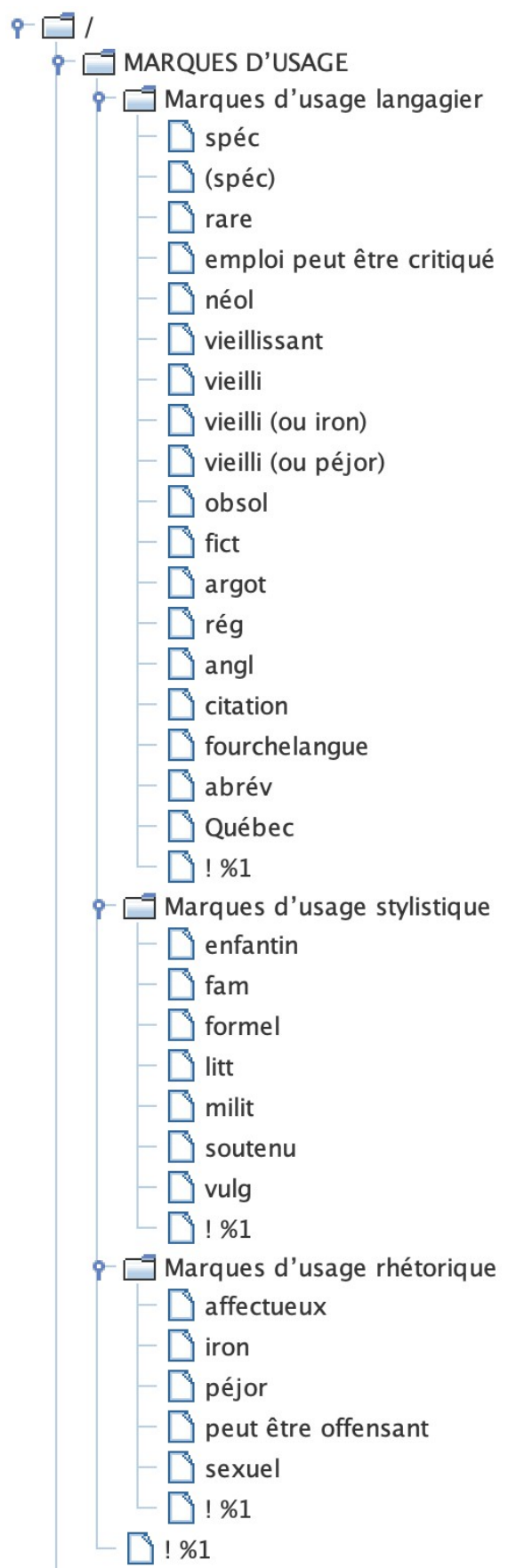

